\title{
Estimation of Residential Radon Concentration in Pennsylvania Counties by Data Fusion
}

\author{
Xuze Zhang ${ }^{1}$, Saumyadipta Pyne ${ }^{2}$, and Benjamin Kedem ${ }^{1}$ \\ ${ }^{1}$ Department of Mathematics and Institute for Systems \\ Research, University of Maryland, College Park \\ ${ }^{2}$ Public Health Dynamics Laboratory, and Department of \\ Biostatistics, Graduate School of Public Health, University of \\ Pittsburgh, Pittsburgh
}

December 18, 2019 


\begin{abstract}
A data fusion method for the estimation of residential radon level distribution in any Pennsylvania county is proposed. The method is based on a multisample density ratio model with variable tilts and is applied to combined radon data from a reference county of interest and its neighboring counties. Beaver county and its four immediate neighbors are taken as a case in point. The distribution of radon concentration is estimated in each of six periods, and then the analysis is repeated combining the data from all the periods to obtain estimates of Beaver threshold probabilities and the corresponding confidence intervals.
\end{abstract}

Key words: Variable tilt, threshold probabilities, empirical distribution, asymptotic, EPA, real estate. 


\section{Introduction}

Radon-222, or just radon, is a tasteless, colorless and odorless radioactive gas, which is a product of Uranium-238 and Radium-226, both of which are naturally abundant in the soil. Radon is known worldwide as a carcinogen as its inhaled decay products can get trapped in the lung and induce mutations in DNA. It is the leading cause of lung cancer among non-smokers [3]. According to an estimation of the impact of radon-associated lung cancer by the U.S. Environmental Protection Agency (EPA), "out of a total of 157,400 lung cancer deaths nationally in 1995, 21,000 (13.4\%) were radon related. Among non-smokers, an estimated $26 \%$ were radon related" [2]. In 1988, radon was formally categorized as a Group 1 Carcinogen (Human Carcinogen) by the International Agency for Research on Cancer [3]. Many countries now pay more attention to this challenge not only as an occupational hazard to miners, but also as a serious risk factor to the health of the general population resulting from indoor residential exposure via cracks in basement floors, walls, etc. [8]. Building regulations in radon affected areas have been introduced in countries such as the United Kingdom. In 1986, the EPA set an action level (the level above which mitigation is recommended) for residential radon to 4 picocuries per liter $(\mathrm{pCi} / \mathrm{L})$. Yet, approximately 1 in 15 residences in the U.S. may have radon levels higher than the action level. Although mitigation of radon can be achieved effectively through ventilation and suitable building materials, elevated radon levels are of concern to buyers and renters and can therefore impact real estate values.

Historically, residences in certain regions of the state of Pennsylvania (PA) have recorded relatively high radon concentrations. For example, the counties that overlie Reading Prong in southeastern PA were found to have elevated residential radon concentrations [5]. Approximately 40 percent of Pennsylvania homes have radon levels above the EPA action guideline of $4 \mathrm{pCi} / \mathrm{L}$ [1]. In the present study, we used county-level indoor radon concentrations based on records collected between 1989 and 2017 from Beaver county and its neighboring counties in PA. Most of the records were reported to the PA Department of Environmental Protection (PA DEP), Bureau of Radiation Protection, Radon Division, during real estate transactions, as submitted by certified test companies, laboratories, or real estate owners.

In this paper, we show how to estimate the radon concentration distribution in any PA county of interest by combining or fusing the county's radon data with the data from its neighboring counties, using the so called density 
ratio model (DRM) with variable tilts. Such flexibility mitigates the problem of misspecified DRM, and is a new feature of the paper.

Our data consist of radon concentration in Pennsylvania's 67 counties over 6 periods, but as a case in point we shall focus on Beaver County and its neighboring counties, Lawrence, Butler, Allegheny, and Washington. We shall refer to the county of interest, Beaver in the present case, as the reference county.

We follow a two-stage procedure. For each reference county we first estimate its radon concentration distribution for each period by DRM, using the combined data from the reference county and its neighbors. In the present case of Beaver and its neighbors, as the radon distributions in each of the six periods behave similarly, we proceeded next to combine the data from the different periods and used the DRM again to get an improved estimate of the radon concentration distribution for the reference county (Beaver). Unlike the present case, when the radon distributions in each of the six periods behave differently we use the results from the last period only. As mentioned, the entire analysis is based on DRM with variable tilts, a novel idea to be clarified in what follows.

\section{Methodology}

\subsection{Data Description}

The following histograms from five Pennsylvania counties depict typical distributions of radon concentration. For a better visualization, the histograms of log-radon concentration are shown in Figure 1. To discern the distribution pattern near 0, the histograms in Figure 2 are constructed from observations less than $40 \mathrm{pCi} / \mathrm{L}$.

From the histograms in Figures 1 and 2 it is observed that the data are positive and right skewed. Experience shows that both the Gamma and Lognormal distributions are possible models for positive right skewed data. However, as the tail behavior is sensitive to distribution assumptions, either choice could lead to a misspecified model. To alleviate this problem, we render the distribution assumption less strict via a density ratio hybrid structure which caters to both distribution models simultaneously. A goodness-of-fit plot in Section 2.5 supports this procedure in the present case of radon data. This graphical goodness-of-fit has been introduced in [13], 


\section{Beaver}
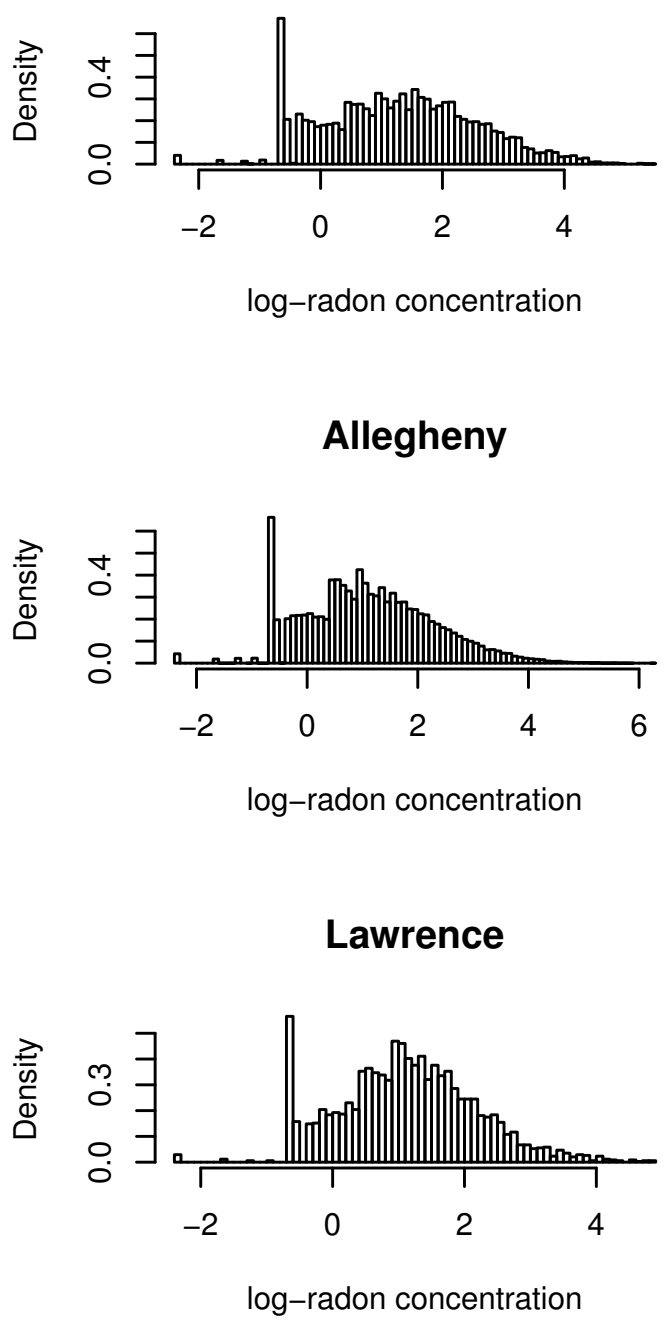
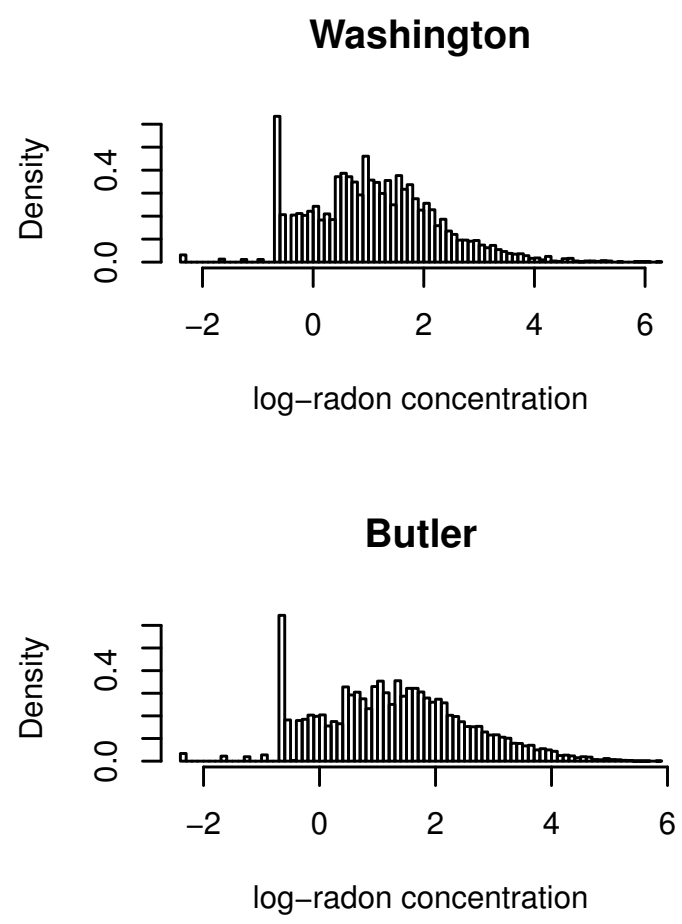

Figure 1: Typical histograms of log-radon concentration from five Pennsylvania counties. 
Beaver
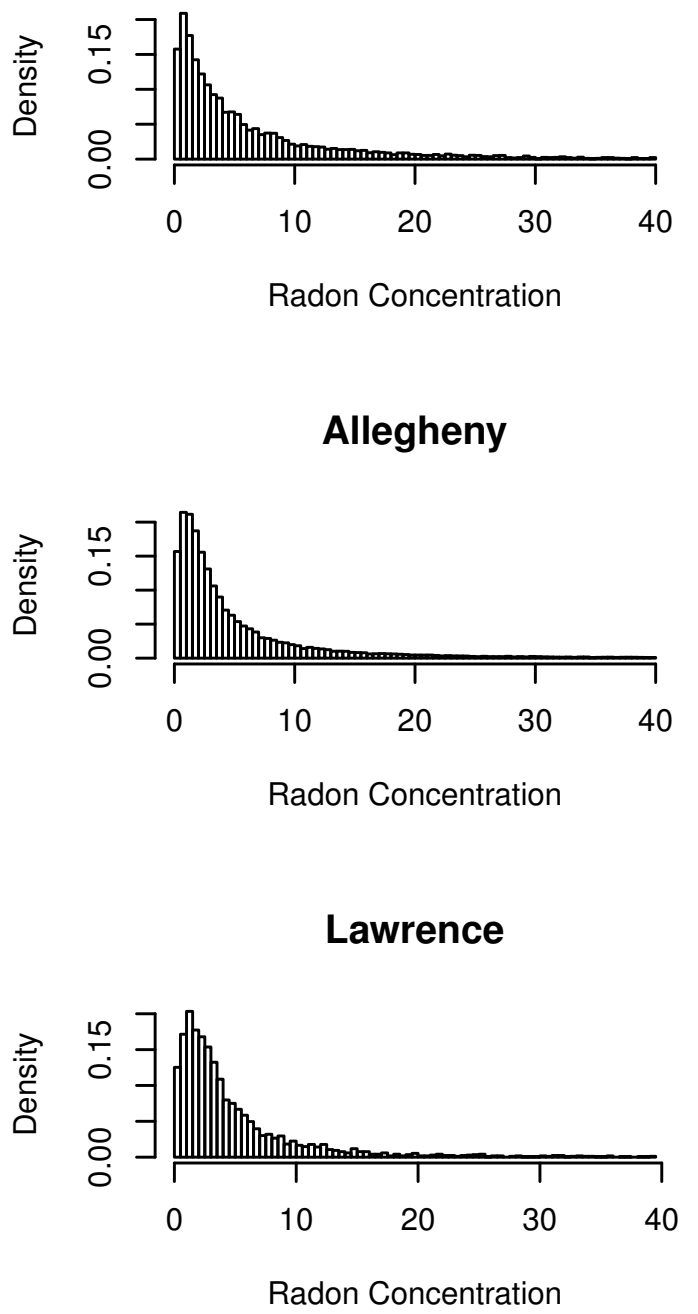

Figure 2: Typical histograms of radon concentration from five Pennsylvania counties truncated at $40 \mathrm{pCi} / \mathrm{L}$.

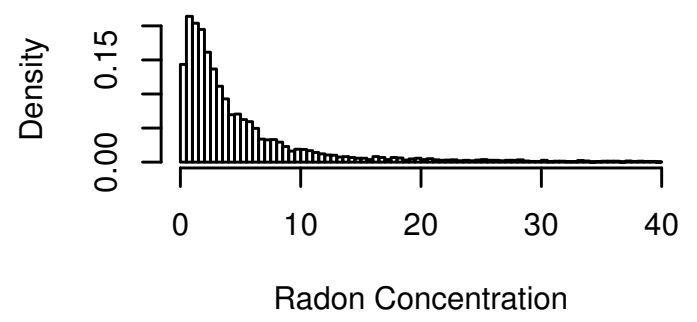

\section{Butler}

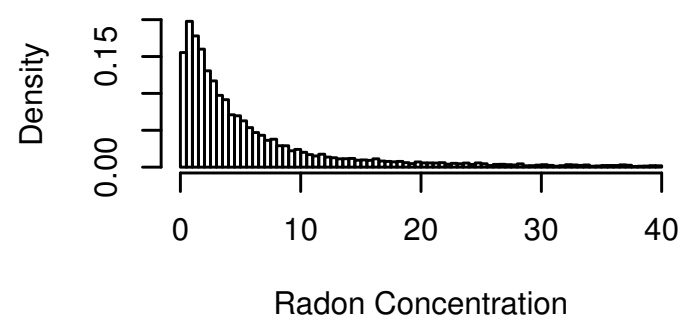




\subsection{Density Ratio Model}

Instead of making particular assumptions about the distributions of radon concentration in each county, we shall estimate the distributions using the following DRM [7], [6], [4], [12] [11],

$$
\frac{g_{k}(x)}{g_{0}(x)}=\exp \left(\alpha_{k}+\boldsymbol{\beta}_{k}^{T} \boldsymbol{h}_{k}(x)\right) \quad k=1, \ldots, m
$$

where $g_{0}(x)$ is the reference radon pdf, $g_{k}(x)$ 's are the pdf's of radon concentration in the neighboring counties, and $m$ denotes the number of neighboring counties. The tilt functions $\boldsymbol{h}_{k}(x)$ 's with respective dimensions $r_{1}, \ldots, r_{m}$ describe the density ratio structure.

We shall first assume the uniform or global tilt $\boldsymbol{h}_{k}(x)=\left(x, \log (x), \log ^{2}(x)\right)^{T}$ for all $k$. Semiparametric inference about the densities and the parameters is then obtained by combining the data of the reference county with that of its neighboring counties. Any of the tilt components $x, \log (x), \log ^{2}(x)$ is removed when the corresponding $\beta$ coefficient is not significantly different from zero. In this sense the density ratio structure is a variable structure, so that different counties could be represented with different tilts relative to the reference.

The tilt $\left(x, \log (x), \log ^{2}(x)\right)^{T}$ is parsimonious in the sense that its dimension is only 3 . However, it has been found sufficient for describing the density ratio structure in the present radon case.

Note that the tilt of two Gamma densities is $(x, \log (x))^{T}$ and the tilt of two Lognormal densities is $\left(\log (x), \log ^{2}(x)\right)^{T}$ so that $\left(x, \log (x), \log ^{2}(x)\right)^{T}$ can represent the density ratio between two Gamma and Lognormal densities. Hence, the assumption of the tilt $\left(x, \log (x), \log ^{2}(x)\right)^{T}$ is less strict in the sense that it allows the distribution to vary between a thin-tailed distribution and a heavy-tailed distribution.

\subsection{Estimation and Asymptotic Result}

More details regarding the theoretical underpinnings of this and the following section are given in the Appendix.

Suppose $\boldsymbol{X}_{0}=\left(X_{01}, \ldots, X_{0 n_{0}}\right)^{T}$ is a sample from the county of interest with size $n_{0}$, and $\boldsymbol{X}_{1}, \ldots, \boldsymbol{X}_{m}$ are the samples from the neighboring counties with sizes $n_{1}, \ldots, n_{m}$, respectively. As above, $\boldsymbol{X}_{0}$ denotes the reference sam- 
ple, and let $G$ be the corresponding reference CDF. Denote the combined or fused sample of size $n=\sum_{k=0}^{m} n_{k}$ by $\boldsymbol{t}=\left(\boldsymbol{X}_{0}^{T}, \ldots, \boldsymbol{X}_{m}^{T}\right)^{T}$.

The parameters are estimated by maximizing the following empirical likelihood

$$
L(\boldsymbol{\alpha}, \boldsymbol{\beta}, G)=\prod_{i=1}^{n} p_{i} \prod_{k=1}^{m} \prod_{j=1}^{n_{k}} w_{k}\left(X_{k j}\right)
$$

subject to the constraints

$$
\sum_{i=1}^{n} p_{i}=1 \quad \sum_{i=1}^{n} p_{i}\left[w_{k}\left(t_{i}\right)-1\right]=0 \quad k=1, \ldots, m
$$

where $p_{i}=d G\left(t_{i}\right)$ and $w_{k}(\cdot)=\exp \left(\alpha_{k}+\boldsymbol{\beta}_{k}^{T} \boldsymbol{h}_{k}(\cdot)\right)$. Denote the parametric estimators by $\tilde{\boldsymbol{\alpha}}, \tilde{\boldsymbol{\beta}}$ and $\tilde{p}_{i}$ 's.

As $n \rightarrow \infty$, the estimators $\tilde{\boldsymbol{\alpha}}, \tilde{\boldsymbol{\beta}}$ are asymptotically normal,

$$
\sqrt{n}\left(\begin{array}{c}
\tilde{\boldsymbol{\alpha}}-\boldsymbol{\alpha}_{0} \\
\tilde{\boldsymbol{\beta}}-\boldsymbol{\beta}_{0}
\end{array}\right) \stackrel{d}{\rightarrow} N(\mathbf{0}, \boldsymbol{\Sigma})
$$

where $\boldsymbol{\alpha}_{0}$ and $\boldsymbol{\beta}_{0}$ are the true parameters. Let $\tilde{G}(t)=\sum_{i=1}^{n} \tilde{p}_{i} I\left[t_{i} \leq t\right]$. Then, following [9] we shall show in the Appendix there are $\Sigma$ and $\sigma(t)$ such that,

$$
\sqrt{n}(\tilde{G}(t)-G(t)) \stackrel{d}{\rightarrow} N(0, \sigma(t))
$$

\subsection{Test of Significance and Tilt Choice}

We start with the uniform or global tilts $\left(x, \log (x), \log ^{2}(x)\right)^{T}$, and then test the significance of the component-functions $x, \log (x), \log ^{2}(x)$, since the density ratio structure could vary between different pairs of counties. Testing the tilt components requires first pairwise DRM fitting using the combined data from the reference and each of its neighbors.

Suppose we combine the data of the reference county and its $i$ 'th neighbor, such that $\boldsymbol{t}=\left(\boldsymbol{X}_{0}^{T}, \boldsymbol{X}_{i}^{T}\right)^{T}$. Based on (4), we have

$$
\sqrt{n}\left(\tilde{\boldsymbol{\beta}}_{i}-\boldsymbol{\beta}_{i}\right) \stackrel{d}{\rightarrow} N\left(\mathbf{0}, \boldsymbol{\Sigma}_{\boldsymbol{\beta}_{i}}\right),
$$

and hence we can test the hypothesis $H_{0}: \boldsymbol{\beta}_{i}=\mathbf{0}$ by the chi-square test

$$
\chi^{2}=n \tilde{\boldsymbol{\beta}}_{i}^{T} \tilde{\boldsymbol{\Sigma}}_{\boldsymbol{\beta}_{i}}^{-1} \tilde{\boldsymbol{\beta}}_{i} \stackrel{d}{\rightarrow} \chi_{\nu}^{2}
$$


where $\nu$ is the dimension of $\boldsymbol{\beta}_{i}$. If we do not reject the hypothesis, then the reference and its neighbor are equidistributed. On the other hand, if the hypothesis is rejected, then we test the significance of each $\beta_{i j}$ from $\boldsymbol{\beta}_{i}=\left(\beta_{i 1}, \ldots, \beta_{i r_{i}}\right)$ using the Z-test defined by

$$
Z=\frac{\tilde{\beta}_{i j}}{\sqrt{\frac{\tilde{\sigma}_{i j}^{2}}{n}}}
$$

where $\tilde{\sigma}_{i j}^{2}$ is the corresponding estimated variance of $\tilde{\beta}_{i j}$. If $\beta_{i j}$ is insignificant, we eliminate the corresponding term in the tilt function to form a reduced tilt function.

\subsection{Refined Model and Goodness-of-Fit}

Once the pairwise tilt functions are refined by the previous preliminary analysis, the density ratio model (1) is fitted again with the modified tilts, using the combined data from the reference county and all its neighbors, either for each period or for all the periods. The Beaver example in the Section 4 clarifies this point. In that way we obtain the estimated reference CDF $\tilde{G}$ and, for any threshold $T$, the estimated threshold probabilities $1-\tilde{G}(T)$ and their asymptotic confidence intervals.

The validity of the density ratio structure with variable tilts can be verified by plotting $\tilde{G}$, an outcome of DRM, versus the model free empirical distribution function $\hat{G}$ obtained from the reference sample only. Such goodnessof-fit plots are useful graphical measures which show the closeness of $\tilde{G}$ to $\hat{G}|13|, \mid 14$.

\section{Simulation}

In this section it is illustrated, by a simulation of a special case, that the estimate of the reference CDF obtained by a DRM with variable tilts is more precise than both of the estimates obtained by a DRM with a global tilt and by the empirical CDF from the reference $\boldsymbol{X}_{0}$ in terms of mean integrated 
absolute error (MIAE) and mean integrated squared error (MISE),

$$
\begin{aligned}
& \text { MIAE }=\mathrm{E} \int|\hat{f}(x)-f(x)| d x \\
& \text { MISE }=\mathrm{E} \int(\hat{f}(x)-f(x))^{2} d x .
\end{aligned}
$$

Here $\hat{f}$ represents the three different estimates $\tilde{G}_{u}, \tilde{G}_{r}$ and $\hat{G}$, and $f$ is the true reference $\mathrm{CDF} G$.

So, consider three random samples $\boldsymbol{X}_{0} \sim \operatorname{Exp}(2), \boldsymbol{X}_{1} \sim \operatorname{Gamma}(2,2)$ and $\boldsymbol{X}_{3} \sim \operatorname{Lognormal}(1,1)$ with size $n_{0}, n_{1}$ and $n_{2}$, respectively. They follow the true density ratio structure

$$
\frac{g_{1}(x)}{g_{0}(x)}=\exp (\log 2+\log (x))
$$

and

$$
\frac{g_{2}(x)}{g_{0}(x)}=\exp \left(-\frac{1}{2}-\log 2 \sqrt{2 \pi}+2 x-\frac{1}{2}\left(\log ^{2}(x)\right)\right)
$$

We constructed two DRM's, one where both $\frac{g_{1}(x)}{g_{0}(x)}$ and $\frac{g_{2}(x)}{g_{0}(x)}$ are with the global ("u") tilt $\left(x, \log (x), \log ^{2}(x)\right)^{T}$, and the other where $\frac{g_{1}(x)}{g_{0}(x)}$ and $\frac{g_{2}(x)}{g_{0}(x)}$ are with the refined ("r") tilts $\boldsymbol{h}_{1}(x)=\log (x)$ and $\boldsymbol{h}_{2}(x)=\left(x, \log ^{2}(x)\right)^{T}$, respectively. We then obtained the corresponding estimated reference CDF's $\tilde{G}_{u}$ and $\tilde{G}_{r}$, from the combined data, as well as the empirical estimate $\hat{G}$ from the reference sample only, and compared them with true reference CDF $G$.

Thus, we follow the steps:

1. Generate random samples $\boldsymbol{X}_{0}^{(i)} \sim \operatorname{Exp}(2), \boldsymbol{X}_{1}^{(i)} \sim \operatorname{Gamma}(2,2)$ and $\boldsymbol{X}_{3}^{(i)} \sim \operatorname{Lognormal}(1,1)$ with size $n_{0}, n_{1}$ and $n_{2}$.

2. Obtain the estimates $\tilde{G}_{u}^{(i)}, \tilde{G}_{r}^{(i)}$ and $\hat{G}^{(i)}$.

3. Repeat 1 and 2 for $i=1, \ldots, I$ 
4. Approximate MIAE and MISE by

$$
\begin{aligned}
& \widehat{\mathrm{MIAE}}=\frac{1}{I} \sum_{i=1}^{I} \delta \sum_{j=1}^{J}\left|\hat{f}\left(M_{1}+j \delta\right)-f\left(M_{1}+j \delta\right)\right| \\
& \widehat{\mathrm{MISE}}=\frac{1}{I} \sum_{i=1}^{I} \delta \sum_{j=1}^{J}\left(\hat{f}\left(M_{1}+j \delta\right)-f\left(M_{1}+j \delta\right)\right)^{2}
\end{aligned}
$$

where $\left(M_{1}, M_{2}\right)$ is the region we integrate over that satisfies $\int_{-\infty}^{\infty} f(x) d x \approx$ $\int_{M_{1}}^{M_{2}} f(x) d x$ and $\delta=\frac{M_{2}-M_{1}}{J}$.

For our simulation we set $I=300, n_{0}=n_{1}=n_{2}=1000,\left(M_{1}, M_{2}\right)=$ $(0,10)$ and $J=1000$. The results shown in Table 1 indicate that the DRM with the refined tilts gives a better estimate of the reference CDF judging by both measures MIAE and MISE.

Table 1: MIAE and MISE for $\tilde{G}_{u}, \tilde{G}_{r}$ and $\hat{G}$

\begin{tabular}{cccc}
\hline Estimate & $\tilde{G}_{u}$ & $\tilde{G}_{r}$ & $\hat{G}$ \\
\hline MIAE & $1.639 \times 10^{-} 2$ & $1.420 \times 10^{-} 2$ & $1.910 \times 10^{-} 2$ \\
MISE & $2.015 \times 10^{-} 4$ & $1.712 \times 10^{-} 4$ & $2.410 \times 10^{-} 4$ \\
\hline
\end{tabular}

\section{Beaver Example}

Beaver county has 4 neighboring counties: Washington, Allegheny, Butler and Lawrence. The radon sample sizes are shown in Table 2.

Table 2: Sample sizes of Beaver, Washington, Allegheny, Butler and Lawrence

\begin{tabular}{cccccc}
\hline & Beaver & Washington & Allegheny & Butler & Lawrence \\
\hline $89-93$ & 816 & 471 & 12328 & 985 & 231 \\
$94-98$ & 913 & 632 & 11982 & 2046 & 476 \\
$99-03$ & 797 & 642 & 6548 & 1192 & 400 \\
$04-08$ & 1209 & 1017 & 7581 & 1891 & 753 \\
$09-13$ & 2064 & 1820 & 12772 & 3142 & 910 \\
$14-17$ & 1626 & 1472 & 8419 & 1995 & 660 \\
Total & 7425 & 6054 & 59630 & 11251 & 3430 \\
\hline
\end{tabular}

We constructed the density ratio model as in (1) with the uniform tilt function $\boldsymbol{h}_{k}(x)=\left(x, \log (x), \log ^{2}(x)\right)^{T}$ for $k=1$-Washington, 2-Allegheny, 3Butler, 4-Lawrence. 
For each period, we first combined the data from Beaver and each of its neighbors (i.e. pairwise fusion) to get in each case a possibly reduced tilt. The possibly reduced tilts are then used in forming the density ratio model for each period, using the combined data from all four counties plus the reference Beaver county. In that way each period gives us the estimated $\tilde{G}$. Observe that, for each period, the empirical CDF $\hat{G}$ is obtained from the reference county (Beaver) only and is model free. The refined tilt functions for each period are given in Table 3.

Table 3: Refined tilts for each county relative to Beaver, in each period. A hyphen "-" indicates that the radon concentration of the indicated county and that of Beaver are identical.

\begin{tabular}{ccccc}
\hline & Washington & Allegheny & Butler & Lawrence \\
\hline $89-93$ & $\left(x, \log ^{2}(x)\right)$ & $\log (x)$ & - & $\left(x, \log ^{2}(x)\right)$ \\
$94-98$ & $\left(x, \log (x), \log ^{2}(x)\right)$ & $\left(x, \log ^{2}(x)\right)$ & $\left(x, \log (x), \log ^{2}(x)\right)$ & $\left(x, \log (x), \log ^{2}(x)\right)$ \\
$99-03$ & $\left(x, \log ^{2}(x)\right)$ & $\left(x, \log (x), \log ^{2}(x)\right)$ & $\left(x, \log ^{2}(x)\right)$ & $(x, \log (x))$ \\
$04-08$ & $\left(x, \log ^{2}(x)\right)$ & $\left(x, \log ^{2}(x)\right)$ & - & $\left(x, \log ^{2}(x)\right)$ \\
$09-13$ & $\left(x, \log (x), \log ^{2}(x)\right)$ & $\left(x, \log (x), \log ^{2}(x)\right)$ & - & $\left(x, \log (x), \log ^{2}(x)\right)$ \\
$14-17$ & $\left(x, \log (x), \log ^{2}(x)\right)$ & $\left(x, \log ^{2}(x)\right)$ & $\left(x, \log ^{2}(x)\right)$ & $\left(x, \log ^{2}(x)\right)$ \\
\hline
\end{tabular}

From the plots (not shown) of $\tilde{G}$ and $\hat{G}$ from all six periods, it is observed that the empirical CDF's $\hat{G}$ are very close to each other and the estimated CDF's $\tilde{G}$ are even more similar. Therefore, it is sensible to combine the entire data from all the counties and all the periods to get an improved estimate of the reference $G$, and hence improved estimates of the tail probabilities $1-G(T)$ for different thresholds $T$.

Thus, we use the combined data from all time periods and all five counties. We used the following variable tilt functions, suggested by first running pairwise fusions, $\boldsymbol{h}_{1}(x)=\boldsymbol{h}_{4}(x)=\left(x, \log (x), \log ^{2}(x)\right)^{T}$ and $\boldsymbol{h}_{2}(x)=\boldsymbol{h}_{3}(x)=$ $\left(x, \log ^{2}(x)\right)^{T}$. From the following goodness-of-fit plot 3 , it is observed that the pairs $(\hat{G}, \tilde{G})$ lie essentially on a $45^{\circ}$-line, pointing to a satisfactory DRM.

Finally, in Table 4, we display Beaver threshold probability estimates for different thresholds $T$ and their corresponding confidence intervals obtained from (54) in the Appendix. Additionally, we also display the empirical estimates and the corresponding confidence intervals to make a comparison with the density ratio estimates. It is readily seen that the confidence intervals obtained from the fused data using $\tilde{G}(T)$ obtained with variable tilts are shorter. Similar analysis can be repeated for any county and its immediate neighbors. 


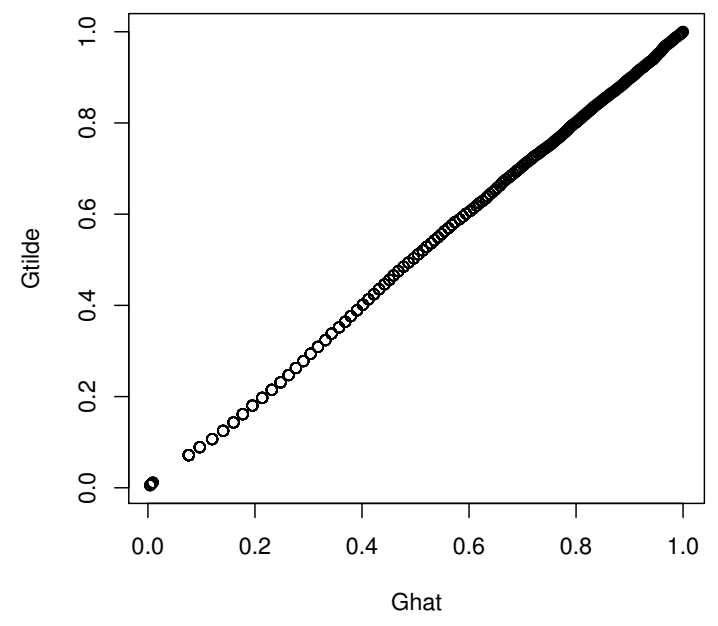

Figure 3: $\tilde{G}$ versus $\hat{G}$

In general, an advantage of using $\tilde{G}$ over $\hat{G}$ is that $\tilde{G}$ is smoother and has a greater support and hence can give more information about the tail of the reference distribution. 
Table 4: Beaver threshold probability estimates and 95\% confidence intervals for threshold $T=5,10,25,50,100,150,200$. The intervals obtained from the fused data using $\tilde{G}(T)$ are shorter.

\begin{tabular}{ccc}
\hline$T$ & $1-\tilde{G}(T)$ & $95 \%$ CI \\
\hline 5 & $3.991 \times 10^{-1}$ & $\left(3.896 \times 10^{-1}, 4.085 \times 10^{-1}\right)$ \\
10 & $2.175 \times 10^{-1}$ & $\left(2.093 \times 10^{-1}, 2.256 \times 10^{-1}\right)$ \\
25 & $7.001 \times 10^{-2}$ & $\left(6.530 \times 10^{-2}, 7.471 \times 10^{-2}\right)$ \\
50 & $1.920 \times 10^{-2}$ & $\left(1.666 \times 10^{-2}, 2.175 \times 10^{-2}\right)$ \\
100 & $2.697 \times 10^{-3}$ & $\left(1.854 \times 10^{-3}, 3.540 \times 10^{-3}\right)$ \\
150 & $5.727 \times 10^{-4}$ & $\left(2.773 \times 10^{-4}, 8.681 \times 10^{-4}\right)$ \\
200 & $1.263 \times 10^{-4}$ & $\left(4.889 \times 10^{-5}, 2.037 \times 10^{-4}\right)$ \\
\hline$T$ & $1-\hat{G}(T)$ & $95 \% \mathrm{CI}$ \\
\hline 5 & $4.057 \times 10^{-1}$ & $\left(3.945 \times 10^{-1}, 4.168 \times 10^{-1}\right)$ \\
10 & $2.183 \times 10^{-1}$ & $\left(2.089 \times 10^{-1}, 2.277 \times 10^{-1}\right)$ \\
25 & $6.667 \times 10^{-2}$ & $\left(6.099 \times 10^{-2}, 7.234 \times 10^{-2}\right)$ \\
50 & $1.980 \times 10^{-2}$ & $\left(1.663 \times 10^{-2}, 2.297 \times 10^{-2}\right)$ \\
100 & $2.559 \times 10^{-3}$ & $\left(1.410 \times 10^{-3}, 3.708 \times 10^{-3}\right)$ \\
150 & $6.734 \times 10^{-4}$ & $\left(8.335 \times 10^{-5}, 1.263 \times 10^{-3}\right)$ \\
200 & $2.693 \times 10^{-4}$ & $\left(-1.039 \times 10^{-4}, 6.426 \times 10^{-4}\right)$ \\
\hline
\end{tabular}

\section{Summary}

The density ratio model and its theoretical underpinnings have been discussed in the context of case-control studies in quite a few works including |7], [4], [9], |10], [12], using fixed tilts. Here we have applied an extension, which uses variable tilts, in the estimation of threshold probabilities of radon concentration, fusing or combining data from several Pennsylvania counties. We have focused on Beaver county and its four immediate neighbors, and relatively high thresholds much beyond the cutoff point of 4 picocurie per liter $(4 \mathrm{pCi} / \mathrm{L})$ [2]. The results in Table 4 using a data fusion method are useful for public health policy and real estate property appraisal. 


\section{Appendix A Appendix: Estimation and Asymp- totic Results}

The estimation and asymptotic results of the two-sample DRM with the tilt function $h(x)=x$ were established in [12] and the analysis of the two-sample case with a general tilt can be found in [15]. An extension to the multi-sample case with an unvaried or uniform tilt is proposed in $[7]$ and a more detailed discussion can be found in [4], [9]. The results in this section are thoroughly examined in [9] under the assumption that the tilt functions are the same for each pair of samples: (Beaver, Washington), (Beaver, Allegheny), (Beaver, Butler), (Beaver, Lawrence). We extend the results in $[9]$ to account for variable tilts.

\section{A.1 Estimation}

Let $\boldsymbol{X}_{0}, \ldots, \boldsymbol{X}_{m}$ be the samples with size $n_{0}, \ldots, n_{m}$ respectively where $\boldsymbol{X}_{0}$ denotes the reference sample. Combine the data and denote it as $\boldsymbol{t}=$ $\left(\boldsymbol{X}_{0}^{T}, \ldots, \boldsymbol{X}_{m}^{T}\right)^{T}$ with size $n=\sum_{k=0}^{m} n_{k}$. Suppose $\boldsymbol{X}_{k} \sim g_{k}$ for $k=0, \ldots, m$ and they have the density ratio structure as (1). Denote the reference CDF as $G$ and let $w_{k}(\cdot)=\exp \left(\alpha_{k}+\boldsymbol{\beta}_{k}^{T} \boldsymbol{h}_{k}(\cdot)\right)$ for $k=1, \ldots, m$. We assume that

$$
\int\left|h_{i}(t)\right| w_{k}(t) d G(t)<\infty \quad \int\left|\boldsymbol{h}_{i}(t) \boldsymbol{h}_{j}^{T}(t)\right| w_{k}(t) d G(t)<\infty
$$

$\forall i, j=1, \ldots, m$ and $k=0, \ldots, m$ where $w_{0}(\cdot) \equiv 1$.

Let $\boldsymbol{\alpha}=\left(\alpha_{1}, \ldots, \alpha_{m}\right)^{T}, \boldsymbol{\beta}=\left(\boldsymbol{\beta}_{1}^{T}, \ldots, \boldsymbol{\beta}_{m}^{T}\right)^{T}$ and $\boldsymbol{\theta}=\left(\boldsymbol{\alpha}^{T}, \boldsymbol{\beta}^{T}\right)^{T}$. Let $p_{i}=d G\left(t_{i}\right)$ for $i=1, \ldots, n$. We wish to obtain the estimators $\tilde{\boldsymbol{\alpha}}, \tilde{\boldsymbol{\beta}}$ and $\tilde{p}_{i}$ 's by maximizing

$$
L(\boldsymbol{\theta}, G)=\prod_{i=1}^{n} p_{i} \prod_{k=1}^{m} \prod_{j=1}^{n_{k}} w_{k}\left(X_{k j}\right)
$$

subject to

$$
\sum_{i=1}^{n} p_{i}=1 \quad \sum_{i=1}^{n} p_{i}\left[w_{k}\left(t_{i}\right)-1\right]=0 \quad k=1, \ldots, m .
$$

Then the objective function with Lagrange multipliers $\lambda_{0}, \ldots, \lambda_{m}$ becomes

$$
\log L(\boldsymbol{\theta}, G)-\lambda_{0}\left(1-\sum_{i=1}^{n} p_{i}\right)-\cdots-\lambda_{m} \sum_{i=1}^{n} p_{i}\left[w_{m}\left(t_{i}\right)-1\right] .
$$


Differentiate the objective function with respect to $p_{i}$ for $i=1, \ldots, n$, we obtain

$$
1+\lambda_{0} p_{i}-\lambda_{1} p_{i}\left[w_{1}\left(t_{i}\right)-1\right]-\cdots-\lambda_{m}\left[w_{m}\left(t_{i}\right)-1\right]=0 .
$$

Sum over $i$, we have $\lambda_{0}=-n$. Replace $\lambda_{0}$ with $-n$ in 15 , we have

$$
p_{i}=\frac{1}{n+\lambda_{1} p_{i}\left[w_{1}\left(t_{i}\right)-1\right]+\cdots+\lambda_{m}\left[w_{m}\left(t_{i}\right)-1\right]}
$$

for $i=1, \ldots, n$. Substitute $(16)$ into $\log L(\boldsymbol{\theta}, G)$, we have

$$
\begin{aligned}
\log L(\boldsymbol{\theta}, G)= & -\sum_{i=1}^{n} \log \left(n+\lambda_{1} p_{i}\left[w_{1}\left(t_{i}\right)-1\right]+\cdots+\lambda_{m}\left[w_{m}\left(t_{i}\right)-1\right]\right) \\
& +\sum_{k=1}^{m}\left(n_{k} \alpha_{k}+\sum_{j=1}^{n_{k}} \boldsymbol{\beta}_{k}^{T} \boldsymbol{h}_{k}\left(X_{k j}\right)\right) .
\end{aligned}
$$

Differentiate $\log L(\boldsymbol{\theta}, G)$ with respect to $\alpha_{k}$ for $k=1, \ldots, m$,

$$
\begin{aligned}
\frac{\partial \log L(\boldsymbol{\theta}, G)}{\partial \alpha_{k}} & =-\sum_{i=1}^{n} \frac{\lambda_{k} w_{k}\left(t_{i}\right)}{n+\lambda_{1} p_{i}\left[w_{1}\left(t_{i}\right)-1\right]+\cdots+\lambda_{m}\left[w_{m}\left(t_{i}\right)-1\right]}+n_{k} \\
& =-\sum_{i=1}^{n} \lambda_{k} p_{i} w_{k}\left(t_{i}\right)+n_{k} \\
& =-\lambda_{k}+n_{k} .
\end{aligned}
$$

Therefore, we obtain $\lambda_{k}=n_{k}$ by equating $\frac{\partial \log L(\boldsymbol{\theta}, G)}{\partial \alpha_{k}}=0$ for $k=1, \ldots, m$. Substitute $\lambda_{k}$ 's into (16) and denote $\rho_{k} \equiv \frac{n_{k}}{n_{0}}$ for $k=0, \ldots, m$, we have

$$
p_{i}=\frac{1}{n_{0} \sum_{k=0}^{m} \rho_{k} w_{k}\left(t_{i}\right)}
$$

Substitute $p_{i}{ }^{\prime}$ s into (17), $\log L(\boldsymbol{\theta}, G)$ reduces to a function of $\boldsymbol{\theta}$ only.

$$
\begin{aligned}
l(\boldsymbol{\theta}) \equiv \log L(\boldsymbol{\theta}, G)= & -\sum_{i=1}^{n} \log \left(n_{0} \sum_{k=0}^{m} \rho_{k} w_{k}\left(t_{i}\right)\right) \\
& +\sum_{k=1}^{m}\left(n_{k} \alpha_{k}+\sum_{j=1}^{n_{k}} \boldsymbol{\beta}_{k}^{T} \boldsymbol{h}_{k}\left(X_{k j}\right)\right) .
\end{aligned}
$$


Then we obtain the estimators $\tilde{\boldsymbol{\theta}}=\left(\tilde{\boldsymbol{\alpha}}^{T}, \tilde{\boldsymbol{\beta}}^{T}\right)^{T}$ via solving the system of equations

$$
\begin{aligned}
& \frac{\partial l(\boldsymbol{\theta})}{\partial \alpha_{k}}=-\sum_{i=1}^{n} \frac{\rho_{k} w_{k}\left(t_{i}\right)}{\sum_{k=0}^{m} \rho_{k} w_{k}\left(t_{i}\right)}+n_{k}=0 \\
& \frac{\partial l(\boldsymbol{\theta})}{\partial \boldsymbol{\beta}_{k}}=-\sum_{i=1}^{n} \frac{\rho_{k} w_{k}\left(t_{i}\right) \boldsymbol{h}_{k}\left(t_{i}\right)}{\sum_{k=0}^{m} \rho_{k} w_{k}\left(t_{i}\right)}+\sum_{j=1}^{n_{k}} \boldsymbol{h}_{k}\left(X_{k j}\right)=\mathbf{0} .
\end{aligned}
$$

Then obtain the estimators $\tilde{p}_{i}$ for $i=1, \ldots, n$ such that

$$
\tilde{p}_{i}=\frac{1}{n_{0} \sum_{k=0}^{m} \rho_{k} \tilde{w}_{k}\left(t_{i}\right)}
$$

where $\tilde{w}_{k}(\cdot)=\exp \left(\tilde{\alpha}_{k}+\tilde{\boldsymbol{\beta}}_{k}^{T} \boldsymbol{h}_{k}(\cdot)\right)$ for $k=1, \ldots, m$ and $\tilde{w}_{0}(\cdot) \equiv 1$. Thus, the estimator of the reference $\mathrm{CDF} G$ is

$$
\tilde{G}(t)=\sum_{i=1}^{n} \tilde{p}_{i} I\left(t_{i} \leq t\right) .
$$

\section{A.2 Asymptotic Distribution of $\tilde{\boldsymbol{\theta}}$}

In this section, we shall establish the strong consistency and the asymptotic normality of $\tilde{\boldsymbol{\theta}}$. Assume that $l(\boldsymbol{\theta})$ is concave and second-order differentiable, the density ratio model is non-degenerate and $\rho_{k}$ 's are fixed as $n \rightarrow 0$. Let $\boldsymbol{\theta}_{0}$ be the true parameter vector. We shall start with obtaining the expectations of the first order derivatives for $k=1, \ldots, m$

$$
\begin{aligned}
\mathrm{E}\left(\frac{\partial l}{\partial \alpha_{k}}\right) & =-\sum_{i=1}^{n} \mathrm{E}\left(\frac{\rho_{k} w_{k}\left(t_{i}\right)}{\sum_{k=0}^{m} \rho_{k} w_{k}\left(t_{i}\right)}\right)+n_{k} \\
& =-\sum_{u=0}^{m} n_{u} \int \frac{\rho_{k} w_{k}(t)}{\sum_{k=0}^{m} \rho_{k} w_{k}(t)} w_{u}(t) d G(t)+n_{k} \\
& =0
\end{aligned}
$$




$$
\begin{aligned}
\mathrm{E}\left(\frac{\partial l}{\partial \boldsymbol{\beta}_{k}}\right)= & -\sum_{i=1}^{n} \mathrm{E}\left(\frac{\rho_{k} w_{k}\left(t_{i}\right) \boldsymbol{h}_{k}\left(t_{i}\right)}{\sum_{k=0}^{m} \rho_{k} w_{k}\left(t_{i}\right)}\right)+\sum_{j=1}^{n_{k}} E \boldsymbol{h}_{k}\left(X_{k j}\right) \\
= & -\sum_{u=0}^{m} n_{u} \int \frac{\rho_{k} w_{k}(t) \boldsymbol{h}_{k}(t)}{\sum_{j=0}^{m} \rho_{j} w_{j}(t)} w_{u}(t) d G(t) \\
& +n_{k} \int w_{k}(t) \boldsymbol{h}_{k}(t) d G(t) \\
= & \mathbf{0} .
\end{aligned}
$$

By the strong law of large number, for $k=1, \ldots, m$,

$$
\begin{aligned}
& \frac{1}{n} \frac{\partial l}{\partial \alpha_{k}}=-\frac{n_{0}}{n} \sum_{u=0}^{m} \rho_{u} \frac{1}{n_{u}} \sum_{j=1}^{n_{u}} \frac{\rho_{k} w_{k}\left(X_{u j}\right)}{\sum_{k=0}^{m} \rho_{k} w_{k}\left(X_{u j}\right)}+\frac{\rho_{k}}{\sum_{k=0}^{m} \rho_{k}} \\
& \stackrel{\text { a.s. }}{\rightarrow}-\frac{1}{\sum_{k=0}^{m} \rho_{k}} \sum_{u=1}^{m} \rho_{u} \int \frac{\rho_{k} w_{k}(t)}{\sum_{k=0}^{m} \rho_{k} w_{k}(t)} w_{u}(t) d G(t)+\frac{\rho_{k}}{\sum_{k=0}^{m} \rho_{k}} \\
&=0 \\
& \frac{1}{n} \frac{\partial l}{\partial \boldsymbol{\beta}_{k}} \stackrel{\text { a.s. }}{\rightarrow}-\frac{1}{\sum_{k=0}^{m} \rho_{k}} \sum_{u=1}^{m} \rho_{u} \int \frac{\rho_{k} w_{k}(t) \boldsymbol{h}_{k}(t)}{\sum_{k=0}^{m} \rho_{k} w_{k}(t)} w_{u}(t) d G(t) \\
& \quad+\frac{\rho_{k}}{\sum_{k=0}^{m} \rho_{k}} \int w_{k}(t) \boldsymbol{h}_{k}(t) d G(t) \\
&=0 .
\end{aligned}
$$

Denote

$$
\begin{aligned}
& l_{1}(\boldsymbol{\theta})=\sum_{i=1}^{n} \log \sum_{k=0}^{m} \rho_{k} w_{k}\left(t_{i}\right) \\
& l_{2}(\boldsymbol{\theta})=\sum_{k=1}^{m}\left(n_{k} \alpha_{k}+\sum_{j=1}^{n_{k}} \boldsymbol{\beta}_{k}^{T} \boldsymbol{h}_{k}\left(X_{k j}\right)\right),
\end{aligned}
$$

and then we have $l=-n \log n_{0}-l_{1}+l_{2}$. Since $\frac{1}{n} \frac{\partial l}{\partial \boldsymbol{\theta}} \stackrel{a . s .}{\rightarrow} \mathbf{0}$, then

$$
\frac{1}{n}\left|\frac{\partial l_{1}}{\partial \boldsymbol{\theta}}-\frac{\partial l_{2}}{\partial \boldsymbol{\theta}}\right| \stackrel{\text { a.s. }}{\rightarrow} \mathbf{0} .
$$


For $l_{1}$, we have

$$
\frac{\partial^{2} l_{1}}{\partial \boldsymbol{\theta} \partial \boldsymbol{\theta}^{T}}=-\frac{\partial^{2} l}{\partial \boldsymbol{\theta} \partial \boldsymbol{\theta}^{T}}
$$

which indicates that $\frac{\partial^{2} l_{1}}{\partial \boldsymbol{\theta} \partial \boldsymbol{\theta}^{T}}$ is positive definite since $l$ is concave and the model is non-degenerate.

For $l_{2}$,

$$
\frac{\partial l_{2}}{\partial \boldsymbol{\theta}}=\left(n_{1}, \ldots, n_{m}, \sum_{j=1}^{n_{1}} \boldsymbol{h}_{1}^{T}\left(X_{1 j}\right), \ldots, \sum_{j=1}^{n_{m}} \boldsymbol{h}_{m}^{T}\left(X_{m j}\right)\right)^{T}
$$

so that $\frac{\partial l_{2}}{\partial \boldsymbol{\theta}}$ is independent of $\boldsymbol{\theta}$ and

$$
l_{2}=\boldsymbol{\theta}^{T} \frac{\partial l_{2}}{\partial \boldsymbol{\theta}}
$$

To prove the strong consistency, it is sufficient to show that the maximum of $l$ is not obtained on the boundary of any closed sphere of $\boldsymbol{\theta}_{0}$ almost surely. We show for sufficiently large $n, \forall \varepsilon, l\left(\boldsymbol{\theta}_{0}\right)>l\left(\boldsymbol{\theta}^{*}\right)$ almost surely for all $\boldsymbol{\theta}^{*} \in$ $\bar{B}\left(\boldsymbol{\theta}_{0}, \varepsilon\right)$.

Expand $\frac{1}{n} l_{1}$ at $\boldsymbol{\theta}_{0}$,

$$
\frac{1}{n} l_{1}\left(\boldsymbol{\theta}^{*}\right)=\frac{1}{n} l_{1}\left(\boldsymbol{\theta}_{0}\right)+\frac{1}{n}\left(\boldsymbol{\theta}^{*}-\boldsymbol{\theta}_{0}\right)^{T} \frac{1}{n} \frac{\partial l_{1}\left(\boldsymbol{\theta}_{0}\right)}{\partial \boldsymbol{\theta}}+\frac{1}{2 n}\left(\boldsymbol{\theta}^{*}-\boldsymbol{\theta}_{0}\right)^{T} \frac{\partial^{2} l_{1}\left(\boldsymbol{\theta}^{* *}\right)}{\partial \boldsymbol{\theta} \partial \boldsymbol{\theta}^{T}}\left(\boldsymbol{\theta}^{*}-\boldsymbol{\theta}_{0}\right)
$$

where $\boldsymbol{\theta}^{* *}$ is between $\boldsymbol{\theta}_{0}$ and $\boldsymbol{\theta}^{*}$. Since that $\frac{\partial^{2} l_{1}}{\partial \boldsymbol{\theta} \partial \boldsymbol{\theta}^{T}}$ is positive definite, then we have

$$
\frac{1}{n} l_{1}\left(\boldsymbol{\theta}^{*}\right)-\frac{1}{n} l_{1}\left(\boldsymbol{\theta}_{0}\right)-\frac{1}{n}\left(\boldsymbol{\theta}^{*}-\boldsymbol{\theta}_{0}\right)^{T} \frac{1}{n} \frac{\partial l_{1}\left(\boldsymbol{\theta}_{0}\right)}{\partial \boldsymbol{\theta}}>0 .
$$

By (28), for sufficiently large $n$,

$$
\frac{1}{n} l_{1}\left(\boldsymbol{\theta}^{*}\right)-\frac{1}{n} l_{1}\left(\boldsymbol{\theta}_{0}\right)-\frac{1}{n}\left(\boldsymbol{\theta}^{*}-\boldsymbol{\theta}_{0}\right)^{T} \frac{1}{n} \frac{\partial l_{2}\left(\boldsymbol{\theta}_{0}\right)}{\partial \boldsymbol{\theta}}>0 .
$$

Since that $\frac{\partial l_{2}}{\partial \boldsymbol{\theta}}$ is independent of $\boldsymbol{\theta}$ and $(31)$, then

$$
\begin{aligned}
\frac{1}{n} l_{1}\left(\boldsymbol{\theta}^{*}\right)-\frac{1}{n} l_{1}\left(\boldsymbol{\theta}_{0}\right)- & \frac{1}{n} l_{2}\left(\boldsymbol{\theta}^{*}\right)+\frac{1}{n} l_{2}\left(\boldsymbol{\theta}_{0}\right)>0 \\
\frac{1}{n} l_{1}\left(\boldsymbol{\theta}_{0}\right)-\frac{1}{n} l_{1}\left(\boldsymbol{\theta}^{*}\right) & >0
\end{aligned}
$$


almost surely. Therefore, the strong consistency of the estimator $\tilde{\boldsymbol{\theta}}$ is established.

Now we wish to obtain the limit of $-\frac{1}{n} \frac{\partial^{2} l}{\partial \boldsymbol{\theta} \partial \boldsymbol{\theta}^{T}}$. By the strong law of large number, for $k, k^{\prime}=1, \ldots, m$ and $k \neq k^{\prime}$, as $n \rightarrow \infty$,

$$
\begin{aligned}
& -\frac{1}{n} \frac{\partial^{2} l}{\partial \alpha_{k}^{2}} \stackrel{a . s .}{\rightarrow} \frac{\rho_{k}}{\sum_{j=0}^{m} \rho_{j}}-\frac{\rho_{k}^{2}}{\sum_{j=0}^{m} \rho_{j}} \int \frac{w_{k}^{2}(t)}{\sum_{j=0}^{m} \rho_{j} w_{j}(t)} d G(t) \\
& -\frac{1}{n} \frac{\partial^{2} l}{\partial \alpha_{k} \partial \alpha_{k^{\prime}}} \stackrel{\text { a.s. }}{\rightarrow}-\frac{\rho_{k} \rho_{k^{\prime}}}{\sum_{j=0}^{m} \rho_{j}} \int \frac{w_{k}(t) w_{k^{\prime}}(t)}{\sum_{j=0}^{m} \rho_{j} w_{j}(t)} d G(t) \\
& -\frac{1}{n} \frac{\partial^{2} l}{\partial \alpha_{k} \partial \boldsymbol{\beta}_{k}^{T}} \stackrel{a . s .}{\rightarrow} \frac{\rho_{k}}{\sum_{j=0}^{m} \rho_{j}} \int w_{k}(t) \boldsymbol{h}_{k}^{T}(t) d G(t) \\
& -\frac{\rho_{k}^{2}}{\sum_{j=0}^{m} \rho_{j}} \int \frac{w_{k}^{2}(t) \boldsymbol{h}_{k}^{T}(t)}{\sum_{j=0}^{m} \rho_{j} w_{j}(t)} d G(t) \\
& -\frac{1}{n} \frac{\partial^{2} l}{\partial \alpha_{k} \partial \boldsymbol{\beta}_{k^{\prime}}^{T}} \stackrel{a . s .}{\rightarrow}-\frac{\rho_{k} \rho_{k^{\prime}}}{\sum_{j=0}^{m} \rho_{j}} \int \frac{w_{k}(t) w_{k^{\prime}}(t) \boldsymbol{h}_{k^{\prime}}^{T}(t)}{\sum_{j=0}^{m} \rho_{j} w_{j}(t)} d G(t) \\
& -\frac{1}{n} \frac{\partial^{2} l}{\partial \boldsymbol{\beta}_{k} \partial \boldsymbol{\beta}_{k}^{T}} \stackrel{a . s .}{\rightarrow} \frac{\rho_{k}}{\sum_{j=0}^{m} \rho_{j}} \int w_{k}(t) \boldsymbol{h}_{k}(t) \boldsymbol{h}_{k}^{T}(t) d G(t) \\
& -\frac{\rho_{k}^{2}}{\sum_{j=0}^{m} \rho_{j}} \int \frac{w_{k}^{2}(t) \boldsymbol{h}_{k}(t) \boldsymbol{h}_{k}^{T}(t)}{\sum_{j=0}^{m} \rho_{j} w_{j}(t)} d G(t) \\
& -\frac{1}{n} \frac{\partial^{2} l}{\partial \boldsymbol{\beta}_{k} \partial \boldsymbol{\beta}_{k^{\prime}}^{T}} \stackrel{a . s .}{\rightarrow}-\frac{\rho_{k} \rho_{k^{\prime}}}{\sum_{j=0}^{m} \rho_{j}} \int \frac{w_{k}(t) w_{k^{\prime}}(t) \boldsymbol{h}_{k}(t) \boldsymbol{h}_{k^{\prime}}^{T}(t)}{\sum_{j=0}^{m} \rho_{j} w_{j}(t)} d G(t) \text {. }
\end{aligned}
$$


Define the following quantities for $k, k^{\prime}=1, \ldots, m$

$$
\begin{aligned}
& A_{k k^{\prime}}=\int \frac{w_{k}(t) w_{k^{\prime}}(t)}{\sum_{j=0}^{m} \rho_{j} w_{j}(t)} d G(t) \\
& \boldsymbol{B}_{k k^{\prime}}=\int \frac{w_{k}(t) w_{k^{\prime}}(t) \boldsymbol{h}_{k^{\prime}}^{T}(t)}{\sum_{j=0}^{m} \rho_{j} w_{j}(t)} d G(t) \\
& \boldsymbol{C}_{k k^{\prime}}=\int \frac{w_{k}(t) w_{k^{\prime}}(t) \boldsymbol{h}_{k}(t) \boldsymbol{h}_{k^{\prime}}^{T}(t)}{\sum_{j=0}^{m} \rho_{j} w_{j}(t)} d G(t) \\
& \boldsymbol{E}_{k}=\int w_{k}(t) \boldsymbol{h}_{k}(t) d G(t) \\
& \overline{\boldsymbol{E}}_{k}=\int w_{k}(t) \boldsymbol{h}_{k}(t) \boldsymbol{h}_{k}^{T}(t) d G(t) \\
& \boldsymbol{V}_{k}=\overline{\boldsymbol{E}}_{k}-\boldsymbol{E}_{k} \boldsymbol{E}_{k}^{T} \\
& \boldsymbol{A}=\left(A_{i j}\right)_{m \times m} \quad \boldsymbol{B}=\left(\boldsymbol{B}_{i j}\right)_{m \times r} \quad \boldsymbol{C}=\left(\boldsymbol{C}_{i j}\right)_{r \times r} \\
& \boldsymbol{\rho}=\left[\begin{array}{ccc}
\rho_{1} & \cdots & 0 \\
\vdots & \ddots & \vdots \\
0 & \cdots & \rho_{m}
\end{array}\right]_{m \times m} \quad \overline{\boldsymbol{\rho}}=\left[\begin{array}{ccc}
\rho_{1} \boldsymbol{I}_{r_{1}} & \cdots & \mathbf{0} \\
\vdots & \ddots & \vdots \\
\mathbf{0} & \cdots & \rho_{m} \boldsymbol{I}_{r_{m}}
\end{array}\right]_{r \times r} \\
& \boldsymbol{E}=\left[\begin{array}{ccc}
\boldsymbol{E}_{1} & \cdots & \mathbf{0} \\
\vdots & \ddots & \vdots \\
\mathbf{0} & \cdots & \boldsymbol{E}_{m}
\end{array}\right]_{r \times m} \quad \overline{\boldsymbol{E}}=\left[\begin{array}{ccc}
\overline{\boldsymbol{E}}_{1} & \cdots & \mathbf{0} \\
\vdots & \ddots & \vdots \\
\mathbf{0} & \cdots & \overline{\boldsymbol{E}}_{m}
\end{array}\right]_{r \times r} \\
& \boldsymbol{V}=\left[\begin{array}{ccc}
\boldsymbol{V}_{1} & \cdots & \mathbf{0} \\
\vdots & \ddots & \vdots \\
\mathbf{0} & \cdots & \boldsymbol{V}_{m}
\end{array}\right]_{r \times r}
\end{aligned}
$$

where $r_{k}$ is the dimension of $\boldsymbol{h}_{k}$ and $r=\sum_{k=1}^{m} r_{k}$. Therefore,

$$
-\frac{1}{n} \frac{\partial^{2} l}{\partial \boldsymbol{\theta} \partial \boldsymbol{\theta}^{T}} \stackrel{\text { a.s. }}{\rightarrow} \boldsymbol{S}=\frac{1}{\sum_{k=0}^{m} \rho_{k}}\left[\begin{array}{ll}
\boldsymbol{S}_{11} & \boldsymbol{S}_{12} \\
\boldsymbol{S}_{12}^{T} & \boldsymbol{S}_{22}
\end{array}\right]
$$

where

$$
\begin{aligned}
\boldsymbol{S}_{11} & =\boldsymbol{\rho}-\boldsymbol{\rho} \boldsymbol{A} \boldsymbol{\rho} \\
\boldsymbol{S}_{12} & =\boldsymbol{\rho} \boldsymbol{E}^{T}-\boldsymbol{\rho} \boldsymbol{B} \overline{\boldsymbol{\rho}} \\
\boldsymbol{S}_{22} & =\overline{\boldsymbol{\rho}} \overline{\boldsymbol{E}}-\overline{\boldsymbol{\rho}} \boldsymbol{C} \overline{\boldsymbol{\rho}} .
\end{aligned}
$$


Next, obtain the expression of $\operatorname{Var}\left(\frac{1}{\sqrt{n}} \frac{\partial l}{\partial \boldsymbol{\theta}}\right)$. For $k, k^{\prime}=1, \ldots, m$ and $k \neq k^{\prime}$,

$$
\begin{aligned}
& \operatorname{Var}\left(\frac{1}{\sqrt{n}} \frac{\partial l}{\partial \alpha_{k}}\right)=\frac{\rho_{k}^{2}}{\sum_{j=0}^{m} \rho_{j}}\left(A_{k k}-\sum_{j=1}^{m} \rho_{j} A_{k j}^{2}-\left(1-\sum_{j=1}^{m} \rho_{j} A_{k j}\right)^{2}\right) \\
& \operatorname{Cov}\left(\frac{1}{\sqrt{n}} \frac{\partial l}{\partial \alpha_{k}}, \frac{1}{\sqrt{n}} \frac{\partial l}{\partial \alpha_{k^{\prime}}}\right)=\frac{\rho_{k} \rho_{k^{\prime}}}{\sum_{j=0}^{m} \rho_{j}}\left(A_{k k^{\prime}}-\sum_{j=1}^{m} \rho_{j} A_{k j} A_{k^{\prime} j}\right. \\
& \left.-\left(1-\sum_{j=1}^{m} \rho_{j} A_{k j}\right)\left(1-\sum_{j=1}^{m} \rho_{j} A_{k^{\prime} j}\right)\right) \\
& \operatorname{Cov}\left(\frac{1}{\sqrt{n}} \frac{\partial l}{\partial \alpha_{k}}, \frac{1}{\sqrt{n}} \frac{\partial l}{\partial \boldsymbol{\beta}_{k}}\right)=\frac{\rho_{k}^{2}}{\sum_{j=0}^{m} \rho_{j}}\left(A_{k k} \boldsymbol{E}_{k}-\sum_{j=1}^{m} \rho_{j} A_{k j} \boldsymbol{B}_{j k}^{T}\right. \\
& \left.-\left(1-\sum_{j=1}^{m} \rho_{j} A_{k j}\right)\left(\boldsymbol{E}_{k}-\sum_{j=1}^{m} \rho_{j} \boldsymbol{B}_{j k}^{T}\right)\right) \\
& \operatorname{Cov}\left(\frac{1}{\sqrt{n}} \frac{\partial l}{\partial \alpha_{k}}, \frac{1}{\sqrt{n}} \frac{\partial l}{\partial \boldsymbol{\beta}_{k^{\prime}}}\right)=\frac{\rho_{k} \rho_{k^{\prime}}}{\sum_{j=0}^{m} \rho_{j}}\left(A_{k k^{\prime}} \boldsymbol{E}_{k^{\prime}}-\sum_{j=1}^{m} \rho_{j} A_{k j} \boldsymbol{B}_{j k^{\prime}}^{T}\right. \\
& \left.-\left(1-\sum_{j=1}^{m} \rho_{j} A_{k j}\right)\left(\boldsymbol{E}_{k^{\prime}}-\sum_{j=1}^{m} \rho_{j} \boldsymbol{B}_{j k^{\prime}}^{T}\right)\right) \\
& \operatorname{Var}\left(\frac{1}{\sqrt{n}} \frac{\partial l}{\partial \boldsymbol{\beta}_{k}}\right)=\frac{\rho_{k}^{2}}{\sum_{j=0}^{m} \rho_{j}}\left(-\boldsymbol{C}_{k k}-\sum_{j=1}^{m} \rho_{j} \boldsymbol{B}_{j k}^{T} \boldsymbol{B}_{j k}-\left(\boldsymbol{E}_{k}-\sum_{j=1}^{m} \rho_{j} \boldsymbol{B}_{j k}^{T}\right)\right. \\
& \left.\left(\boldsymbol{E}_{k}^{T}-\sum_{j=1}^{m} \rho_{j} \boldsymbol{B}_{j k}\right)+2 \boldsymbol{B}_{k k}^{T} \boldsymbol{E}_{k}^{T}\right)+\frac{\rho_{k}}{\sum_{j=0}^{m} \rho_{j}} \boldsymbol{V}_{k} \\
& \operatorname{Cov}\left(\frac{1}{\sqrt{n}} \frac{\partial l}{\partial \boldsymbol{\beta}_{k}}, \frac{1}{\sqrt{n}} \frac{\partial l}{\partial \boldsymbol{\beta}_{k^{\prime}}}\right)=\frac{\rho_{k} \rho_{k^{\prime}}}{\sum_{j=0}^{m} \rho_{j}}\left(-\boldsymbol{C}_{k k^{\prime}}-\sum_{j=1}^{m} \rho_{j} \boldsymbol{B}_{j k}^{T} \boldsymbol{B}_{j k^{\prime}}\right. \\
& -\left(\boldsymbol{E}_{k}-\sum_{j=1}^{m} \rho_{j} \boldsymbol{B}_{j k}^{T}\right)\left(\boldsymbol{E}_{k^{\prime}}^{T}-\sum_{j=1}^{m} \rho_{j} \boldsymbol{B}_{j k^{\prime}}\right) \\
& \left.+\boldsymbol{B}_{k k^{\prime}}^{T} \boldsymbol{E}_{k}^{T}+\boldsymbol{B}_{k k^{\prime}}^{T} \boldsymbol{E}_{k^{\prime}}^{T}\right) \text {. }
\end{aligned}
$$


Let $\Lambda \equiv \operatorname{Var}\left(\frac{1}{\sqrt{n}} \frac{\partial l}{\partial \boldsymbol{\theta}}\right)$ and $\Lambda \equiv \frac{1}{\sum_{k=0}^{m} \rho_{k}}\left[\begin{array}{cc}\boldsymbol{\Lambda}_{11} & \boldsymbol{\Lambda}_{12} \\ \boldsymbol{\Lambda}_{12}^{T} & \boldsymbol{\Lambda}_{22}\end{array}\right]$, then we have

$$
\begin{aligned}
\boldsymbol{\Lambda}_{11}= & \boldsymbol{\rho}\left(\boldsymbol{A}-\boldsymbol{A} \boldsymbol{\rho} \boldsymbol{A}-\left(\boldsymbol{I}_{m}-\boldsymbol{A} \boldsymbol{\rho}\right) \boldsymbol{J}_{m}\left(\boldsymbol{I}_{m}-\boldsymbol{\rho} \boldsymbol{A}\right)\right) \boldsymbol{\rho} \\
= & \boldsymbol{S}_{11}-\boldsymbol{S}_{11}\left(\boldsymbol{J}_{m}+\boldsymbol{\rho}^{-1}\right) \boldsymbol{S}_{11} \\
\boldsymbol{\Lambda}_{12}= & \boldsymbol{\rho}\left(\boldsymbol{A} \boldsymbol{E}^{T}-\boldsymbol{A} \boldsymbol{\rho} \boldsymbol{B}-\left(\boldsymbol{I}_{m}-\boldsymbol{A} \boldsymbol{\rho}\right) \boldsymbol{J}_{m}\left(\boldsymbol{E}^{T}-\boldsymbol{\rho} \boldsymbol{B}\right)\right) \overline{\boldsymbol{\rho}} \\
= & \boldsymbol{S}_{12}-\boldsymbol{S}_{11}\left(\boldsymbol{J}_{m}+\boldsymbol{\rho}^{-1}\right) \boldsymbol{S}_{12} \\
\boldsymbol{\Lambda}_{22}= & \overline{\boldsymbol{\rho}}\left(-\boldsymbol{C}-\boldsymbol{B}^{T} \boldsymbol{\rho} \boldsymbol{B}-\left(\boldsymbol{E}-\boldsymbol{B}^{T} \boldsymbol{\rho}\right) \boldsymbol{J}_{m}\left(\boldsymbol{E}^{T}-\boldsymbol{\rho} \boldsymbol{B}\right)+\boldsymbol{B}^{T} \boldsymbol{E}^{T}\right. \\
& +\boldsymbol{E} \boldsymbol{B}) \overline{\boldsymbol{\rho}}+\overline{\boldsymbol{\rho}}\left(\overline{\boldsymbol{E}}-\boldsymbol{E} \boldsymbol{E}^{T}\right) \\
= & \boldsymbol{S}_{22}-\boldsymbol{S}_{21}\left(\boldsymbol{J}_{m}+\boldsymbol{\rho}^{-1}\right) \boldsymbol{S}_{12}
\end{aligned}
$$

so that

$$
\boldsymbol{\Sigma} \equiv \boldsymbol{S}^{-1} \boldsymbol{\Lambda} \boldsymbol{S}^{-1}=\boldsymbol{S}^{-1}-\sum_{j=0}^{m} \rho_{j}\left[\begin{array}{cc}
\boldsymbol{J}_{m}+\boldsymbol{\rho}^{-1} & \mathbf{0} \\
\mathbf{0} & \mathbf{0}
\end{array}\right]
$$

Expand $\frac{\partial l}{\partial \boldsymbol{\theta}}$ at $\boldsymbol{\theta}_{0}$ and plug in $\tilde{\boldsymbol{\theta}}$

$$
\begin{aligned}
\frac{\partial l(\tilde{\boldsymbol{\theta}})}{\partial \boldsymbol{\theta}} & =\frac{\partial l\left(\boldsymbol{\theta}_{0}\right)}{\partial \boldsymbol{\theta}}+\frac{\partial^{2} l\left(\boldsymbol{\theta}_{0}\right)}{\partial \boldsymbol{\theta} \partial \boldsymbol{\theta}^{T}}\left(\tilde{\boldsymbol{\theta}}-\boldsymbol{\theta}_{0}\right)+o\left(\left\|\tilde{\boldsymbol{\theta}}-\boldsymbol{\theta}_{0}\right\|\right) \\
\sqrt{n}\left(\tilde{\boldsymbol{\theta}}-\boldsymbol{\theta}_{0}\right) & =-\left(\frac{1}{n} \frac{\partial^{2} l\left(\boldsymbol{\theta}_{0}\right)}{\partial \boldsymbol{\theta} \partial \boldsymbol{\theta}^{T}}\right)^{-1}\left(\frac{1}{\sqrt{n}} \frac{\partial l\left(\boldsymbol{\theta}_{0}\right)}{\partial \boldsymbol{\theta}}\right)+o(1) .
\end{aligned}
$$

By the central limit theorem,

$$
\frac{1}{\sqrt{n}} \frac{\partial l\left(\boldsymbol{\theta}_{0}\right)}{\partial \boldsymbol{\theta}} \stackrel{d}{\rightarrow} N(\mathbf{0}, \boldsymbol{\Lambda}) .
$$

And since $-\frac{1}{n} \frac{\partial^{2} l\left(\boldsymbol{\theta}_{0}\right)}{\partial \boldsymbol{\theta} \partial \boldsymbol{\theta}^{T}} \stackrel{\text { a.s. }}{\rightarrow} \boldsymbol{S}$, by the Slutsky's theorem,

$$
\sqrt{n}\left(\tilde{\boldsymbol{\theta}}-\boldsymbol{\theta}_{0}\right) \stackrel{d}{\rightarrow} N(\mathbf{0}, \boldsymbol{\Sigma})
$$

Hence, the asymptotic normality of $\tilde{\boldsymbol{\theta}}$ is established.

\section{A.3 Asymptotic Behavior of $\tilde{G}$}

In this section, we wish to show that $\sqrt{n}(\tilde{G}-G)$ converges weakly to a zero-mean Gaussian process in $D[-\infty, \infty]$ with covariance function $R$. The proof in [9] can be adopted with a slight modification. To prove the weak 
convergence of $\sqrt{n}(\tilde{G}-G)$, it is sufficient to show the weak convergence of $\sqrt{n}(\tilde{G}-\hat{G})$ due to the weak convergence of the empirical process $\sqrt{n}(\hat{G}-G)$.

Let

$$
\begin{aligned}
& A_{k}(t)=\int \frac{w_{k}(y) I[y \leq t]}{\sum_{j=0}^{m} \rho_{j} w_{j}(y)} d G(y) \\
& \boldsymbol{B}_{k}(t)=\int \frac{w_{k}(y) \boldsymbol{h}_{k}(y) I[y \leq t]}{\sum_{j=0}^{m} \rho_{j} w_{j}(y)} d G(y) \\
& \overline{\boldsymbol{A}}(t)=\left(A_{1}(t), \cdots, A_{m}(t)\right)^{T} \\
& \overline{\boldsymbol{B}}(t)=\left(\boldsymbol{B}_{1}^{T}(t), \cdots, \boldsymbol{B}_{m}^{T}(t)\right)^{T} \\
& H_{1}(t ; \boldsymbol{\theta})=\frac{1}{n_{0}} \sum_{i=1}^{n} \frac{I\left[t_{i} \leq t\right]}{\sum_{j=0}^{m} \rho_{j} w_{j}\left(t_{i}\right)} \\
& H_{2}(t ; \boldsymbol{\theta})=\frac{1}{n}\left(\overline{\boldsymbol{A}}^{T}(t) \boldsymbol{\rho}, \overline{\boldsymbol{B}}^{T}(t) \overline{\boldsymbol{\rho}}\right) \boldsymbol{S}^{-1} \frac{\partial l(\boldsymbol{\theta})}{\partial \boldsymbol{\theta}} .
\end{aligned}
$$

Then we have

$$
\mathrm{E}\left(\frac{\partial H_{1}\left(t ; \boldsymbol{\theta}_{0}\right)}{\partial \boldsymbol{\theta}}\right)=\left(\overline{\boldsymbol{A}}^{T}(t) \boldsymbol{\rho}, \overline{\boldsymbol{B}}^{T} \overline{\boldsymbol{\rho}}\right)^{T}
$$

and by the strong law of large number, we can show

$$
\frac{\partial H_{1}\left(t ; \boldsymbol{\theta}_{0}\right)}{\partial \boldsymbol{\theta}} \stackrel{\text { a.s. }}{\rightarrow} \mathrm{E}\left(\frac{\partial H_{1}\left(t ; \boldsymbol{\theta}_{0}\right)}{\partial \boldsymbol{\theta}}\right) .
$$

By the boundness of $\frac{w_{k}(y)}{\sum_{j=0}^{m} \rho_{j} w_{j}(y)}$ and $\frac{w_{k}(y) \boldsymbol{h}_{k}(y)}{\sum_{j=0}^{m} \rho_{j} w_{j}(y)}$, we have

$$
\sup _{-\infty<t<\infty}\left\|\frac{\partial H_{1}\left(t ; \boldsymbol{\theta}_{0}\right)}{\partial \boldsymbol{\theta}}-\mathrm{E}\left(\frac{\partial H_{1}\left(t ; \boldsymbol{\theta}_{0}\right)}{\partial \boldsymbol{\theta}}\right)\right\| \stackrel{\text { a.s. }}{\rightarrow} 0 .
$$

Expand $\tilde{G}$ at $\boldsymbol{\theta}_{0}$,

$$
\begin{aligned}
\tilde{G}(t)= & H_{1}\left(t ; \boldsymbol{\theta}_{0}\right)+\left(\frac{\partial H_{1}\left(t ; \boldsymbol{\theta}_{0}\right)}{\partial \boldsymbol{\theta}}-\mathrm{E}\left(\frac{\partial H_{1}\left(t ; \boldsymbol{\theta}_{0}\right)}{\partial \boldsymbol{\theta}}\right)\right)^{T}\left(\tilde{\boldsymbol{\theta}}-\boldsymbol{\theta}_{0}\right) \\
& -H_{2}\left(t ; \boldsymbol{\theta}_{0}\right)-\left(\overline{\boldsymbol{A}}^{T}(t) \boldsymbol{\rho}, \overline{\boldsymbol{B}}^{T}(t) \overline{\boldsymbol{\rho}}\right)\left(\boldsymbol{\theta}_{0}-\frac{1}{n} \boldsymbol{S}^{-1} \frac{\partial l\left(\boldsymbol{\theta}_{0}\right)}{\partial \boldsymbol{\theta}}\right) \\
& +o\left(\left\|\tilde{\boldsymbol{\theta}}-\boldsymbol{\theta}_{0}\right\|\right) \\
= & H_{1}\left(t ; \boldsymbol{\theta}_{0}\right)+o\left(\frac{1}{\sqrt{n}}\right)-H_{2}\left(t ; \boldsymbol{\theta}_{0}\right)+o\left(\frac{1}{\sqrt{n}}\right)+o\left(\frac{1}{\sqrt{n}}\right) \\
= & H_{1}\left(t ; \boldsymbol{\theta}_{0}\right)-H_{2}\left(t ; \boldsymbol{\theta}_{0}\right)+o\left(\frac{1}{\sqrt{n}}\right) .
\end{aligned}
$$


Hence, $\sqrt{n}(\tilde{G}-\hat{G})$ can be approximated by $\sqrt{n}\left(H_{1}-H_{2}-\hat{G}\right)$ uniformly in $t$. It is left to show that the finite dimension distribution of $\sqrt{n}\left(H_{1}-H_{2}-\hat{G}\right)$ converges and $\sqrt{n}\left(H_{1}-H_{2}-\hat{G}\right)$ is tight. By the same derivation in the proof of the Lemma 3.5 in $[9], \forall k$ and $t_{1}, \ldots, t_{k}$,

$$
\sqrt{n}\left(H_{1}\left(t_{1}\right)-H_{2}\left(t_{1}\right)-\hat{G}\left(t_{1}\right), \ldots, H_{1}\left(t_{k}\right)-H_{2}\left(t_{k}\right)-\hat{G}\left(t_{k}\right)\right)^{T} \stackrel{d}{\rightarrow} N(\mathbf{0}, \boldsymbol{\Delta})
$$

where $\Delta$ is the covariance matrix depending on the covariance function

$$
\begin{aligned}
& \operatorname{Cov}\left(\sqrt{n}\left(H_{1}(s)-H_{2}(s)-\hat{G}(s)\right), \sqrt{n}\left(H_{1}(t)-H_{2}(t)-\hat{G}(t)\right)\right) \\
= & \sum_{j=0}^{m} \rho_{j} \sum_{j=1}^{m} \rho_{j} A_{j}(s \wedge t)-\left(\overline{\boldsymbol{A}}^{T}(t) \boldsymbol{\rho}, \overline{\boldsymbol{B}}^{T}(t) \overline{\boldsymbol{\rho}}\right) \boldsymbol{S}^{-1}\left(\overline{\boldsymbol{A}}^{T}(s) \boldsymbol{\rho}, \overline{\boldsymbol{B}}^{T}(s) \overline{\boldsymbol{\rho}}\right)^{T} .
\end{aligned}
$$

Also, the tightness of $\sqrt{n}\left(H_{1}-\hat{G}\right)$ and $\sqrt{n} H_{2}$ can be proved by Lemma 3.6 and Lemma 3.7 in $\mid 9]$. Therefore, we have shown that $\sqrt{n}(\tilde{G}-G)$ converges weakly to a zero-mean Gaussian process in $D[-\infty, \infty]$. By the same derivation in the proof of the Theorem 3.9 in [9], we finally have

$$
\sqrt{n}(\tilde{G}(t)-G(t)) \stackrel{d}{\rightarrow} N(0, \sigma(t))
$$

where

$$
\begin{aligned}
\sigma(t)= & \left(\sum_{j=0}^{m} \rho_{j}\right)\left(G(t)-G^{2}(t)-\sum_{j=1}^{m} \rho_{j} A_{j}(t)\right) \\
& +\left(\overline{\boldsymbol{A}}^{T}(t) \boldsymbol{\rho}, \overline{\boldsymbol{B}}^{T}(t) \overline{\boldsymbol{\rho}}\right) \boldsymbol{S}^{-1}\left(\overline{\boldsymbol{A}}^{T}(t) \boldsymbol{\rho}, \overline{\boldsymbol{B}}^{T}(t) \overline{\boldsymbol{\rho}}\right)^{T}
\end{aligned}
$$




\section{References}

[1] https://www.dep.pa.gov/Business/RadiationProtection/ RadonDivision/Pages/Radon-in-thehome.aspx. PA Department of Environmental Protection.

[2] EPA Assessment of Risks from Radon in Homes: EPA 402-R03-003. https://www.epa.gov/sites/production/files/2015-05/ documents/402-r-03-003.pdf. U.S. Environmental Protection Agency.

[3] Indoor radon: A public health perspective, Int. J. Environ. Stud, 67 (2009), p. 108. World Health Organization.

[4] K. Benjamin, O. V. De, and S. Michael, Statistical Data Fusion, World Scientific, 2017.

[5] J. A. Casey, E. L. Ogburn, S. G. Rasmussen, J. K. Irving, J. Pollak, P. A. Locke, And B. S. Schwartz, Predictors of indoor radon concentrations in Pennsylvania, 1989-2013, Environmental health perspectives, 123 (2015), pp. 1130-1137.

[6] K. FoKIAnos, Merging information for semiparametric density estimation, Journal of the Royal Statistical Society: Series B (Statistical Methodology), 66 (2004), pp. 941-958.

[7] K. Fokinnos, B. Kedem, J. Qin, And D. A. Short, A semiparametric approach to the one-way layout, Technometrics, 43 (2001), pp. 56-65.

[8] S. Keith, J. Doyle, C. Harper, M. Mumtaz, O. Tarrago, D. Wohlers, G. Diamond, M. Citra, And L. Barber, Toxicological profile for radon, (2012).

[9] G. Lu, Asymptotic Theory for Multiple-Sample Semiparametric Density Ratio Models and its Application to Mortality Forecasting, (2007). Ph.D. Dissertation, University of Maryland, College Park.

[10] J. QIN, Biased Sampling, Over-identified Parameter Problems and Beyond, Springer, 2017.

[11] J. QIN AND J. LAWLESS, Empirical likelihood and general estimating equations, The Annals of Statistics, (1994), pp. 300-325. 
[12] J. QIN AND B. ZHANG, A goodness-of-fit test for logistic regression models based on case-control data, Biometrika, 84 (1997), pp. 609-618.

[13] A. Voulgaraki, Semiparametric Regression and Mortality Rate Prediction, (2011). Ph.D. Dissertation, University of Maryland, College Park.

[14] A. Voulgaraki, B. Kedem, B. I. Graubard, et Al., Semiparametric regression in testicular germ cell data, The Annals of Applied Statistics, 6 (2012), pp. 1185-1208.

[15] B. ZHANG, A goodness of fit test for multiplicative-intercept risk models based on case-control data, Statistica Sinica, (2000), pp. 839-865. 